\title{
The Effects of Resource Limitation on a Predator-Prey Model with Control Measures as Nonlinear Pulses
}

\author{
Wenjie Qin, ${ }^{1}$ Sanyi Tang, ${ }^{1}$ and Robert A. Cheke ${ }^{2}$ \\ ${ }^{1}$ College of Mathematics and Information Science, Shaanxi Normal University, Xian 710062, China \\ ${ }^{2}$ Natural Resources Institute, University of Greenwich at Medway, Kent ME4 4TB, UK
}

Correspondence should be addressed to Wenjie Qin; wenjieqin@hotmail.com

Received 16 October 2013; Accepted 26 January 2014; Published 11 March 2014

Academic Editor: Jui-Sheng Lin

Copyright (C) 2014 Wenjie Qin et al. This is an open access article distributed under the Creative Commons Attribution License, which permits unrestricted use, distribution, and reproduction in any medium, provided the original work is properly cited.

The dynamical behavior of a Holling II predator-prey model with control measures as nonlinear pulses is proposed and analyzed theoretically and numerically to understand how resource limitation affects pest population outbreaks. The threshold conditions for the stability of the pest-free periodic solution are given. Latin hypercube sampling/partial rank correlation coefficients are used to perform sensitivity analysis for the threshold concerning pest extinction to determine the significance of each parameter. Comparing this threshold value with that without resource limitation, our results indicate that it is essential to increase the pesticide's efficacy against the pest and reduce its effectiveness against the natural enemy, while enhancing the efficiency of the natural enemies. Once the threshold value exceeds a critical level, both pest and its natural enemies populations can oscillate periodically. Furthermore, when the pulse period and constant stocking number as a bifurcation parameter, the predator-prey model reveals complex dynamics. In addition, numerical results are presented to illustrate the feasibility of our main results.

\section{Introduction}

It is well known that pest outbreaks often cause serious ecological and economic problems, requiring complex control measures to reduce harm due to insect pests of agriculture and insect vectors of important plant, animal, and human diseases. Such measures include use of a variety of chemical pesticides, biological pesticides, and biological control.

Biological control is the reduction of pest populations by other living organisms, often called natural enemies or beneficial species (see [1-3]). Virtually all pests have some natural enemies, and the key to successful pest control is to identify the pest and its main natural enemies and to release the beneficial insects early when pest levels are low. Chemical control relies mainly on the use of synthetic pesticides to suppress pests. Pesticides are useful because they quickly kill a significant portion of a pest population and they sometimes provide the only feasible method for preventing economic loss. However, pesticide pollution is also recognized as a major health hazard to people and to pest's natural enemies. Also, overuse of a single control tactic is discouraged to avoid or delay the development of resistance by the pest to the control tactic, to minimize damage to nontarget organisms, and to preserve the quality of the environment. Therefore, it is natural to combine biological and chemical controls as components of integrated pest management (IPM).

The concept of IPM was introduced in the late 1950s [4], was widely practised during the 1970s and 1980s [5], and is still often adopted. IPM emphasizes the importance of interactions between pests and its natural enemies and is a long-term management strategy to reduce pests to predetermined economic injury levels, with little cost and minimal effects on the environment. IPM has been shown to be more effective than the traditional methods, such as biological control or chemical control alone, both experimentally $[6,7]$ and theoretically $[8,9]$. These results indicate that different pest control techniques should work together rather than against each other.

In the last few decades, in order to consider the consequences of spraying pesticides and introducing additional predators into a natural predator-pest system, many authors have suggested impulsive differential equations to investigate 
the dynamics of pest control models. For the general theory of impulsive differential equations, see $[10,11]$. Recently, many papers have been devoted to the analysis of mathematical models describing IPM strategies. See, for instance, [12-20] and so on.

The emphasis of IPM is on control, not eradication of the pest, as the latter can be impossible, cost-ineffective, or potentially damaging to the environment. The above traditional predator-prey model with IPM has assumed that the fatality rate of pesticide applications with respect to the pest is constant, which implies that the agricultural resources such as pesticides, labor forces, equipments, and costs are very effective and sufficient for controlling pests. However, in reality, every community or country has an appropriate or limited capacity for pesticides, costs, and so forth. Meanwhile, the wide use of the pesticides will potentially damage the environment. A resource is limiting if changes in its availability affect the population equilibrium level [21], which is a function of individual survival and reproduction. Estimates of demography, therefore, can be linked with estimates of resource availability, as they change through time, to yield insights into the effects of resource limitation [22].

Understanding resource limitation is critical to effective management and conservation of populations; however, resource limitation is difficult to quantify partly because it is a dynamic process [23]. In order to investigate the effect of limited resources on the outbreak of pest populations, a saturation phenomenon of the limited resources is considered. That is, we will study the dynamic behaviors of a predator-prey model with nonlinear pulse controls, which are suitable mathematical models to simulate processes with short-term perturbations during their development [11]. The main purposes of this paper are to construct a simple mathematical model including the features of periodic biological and chemical control for pest control to understand how limited resources affect pest outbreaks since the limited resources and the fatality rate of pesticide applications on the pest could depend on its population's density. Also, in order to investigate the effect of the limited capacity of pesticides, a nonlinear continually differentiable function to characterize the saturation phenomenon of the limited resources is introduced. The model is based on the predatorprey model with generalized Holling II functional response.

The organization of present paper is as follows. In the next section, a Holling II predator-prey model with nonlinear pulse is introduced. In Section 3, by using the method of the differential inequality, qualitative analysis, and the discrete dynamical system determined by the stroboscopic map and Floquet theory, a set of sufficient conditions which guarantee the existence and stability of the pest-free periodic solution are obtained. Meanwhile, we explored the parameter space by performing an uncertainty analysis and sensitivity analysis using the Latin hypercube sampling (LHS) method and evaluating partial rank correlation coefficients (PRCCs) for various input parameters against threshold conditions and then the key factors which are most significantly related to the threshold conditions were determined (see [18, 24, 25]). Section 4 focuses on the sufficient conditions under which the positive periodic solution exists by using the bifurcation theorem. The paper ends with some interesting biological conclusions and numerical bifurcation analyses, which complement the theoretical findings.

\section{Model Formulation}

The predator-prey dynamics are modelled by two ordinary differential equations (see $[26,27]$ ). Based on experiments, Holling [28] suggested three different kinds of functional responses for different kinds of species to model the phenomena of predation, which made the standard Lotka-Volterra model more realistic. So the basic model we consider is based on the following predator-prey model where the prey has logistic growth with no predators and the predator has Holling II functional response:

$$
\begin{gathered}
\dot{x}=a x-b x^{2}-\frac{c x y}{1+\omega x}, \\
\dot{y}=\mu \frac{c x y}{1+\omega x}-d y,
\end{gathered}
$$

where $a, b, c, d, \omega, \mu$ are positive constants. $x(t), y(t)$ are the densities of the prey and predator at time $t$, respectively.

With the idea of impulsive perturbations, motivated by $[29,30]$, a predator-prey model concerning IPM strategies is proposed as follows:

$$
\begin{aligned}
& \dot{x}=a x-b x^{2}-\frac{c x y}{1+\omega x}, \quad t \neq n T, n \in \mathcal{N}, \\
& \dot{y}=\mu \frac{c x y}{1+\omega x}-d y, \\
& x\left(t^{+}\right)=\left(1-p_{1}\right) x, \quad t=n T, n \in \mathcal{N}, \\
& y\left(t^{+}\right)=\left(1-p_{2}\right) y+\sigma,
\end{aligned}
$$

where $\mathcal{N}=\{0,1,2, \ldots\}$ and $T$ is the pulse period. $0 \leq p_{1}<$ $1\left(0 \leq p_{2}<1\right)$ represents the fraction of pests (predators) which die due to the pesticide at $t=n T$, and $\sigma>0$ is the release amount of predator at $t=n T$.

It is assumed that the fatality rate of pesticide applications with respect to the pest (natural enemy) is a constant $p_{1}\left(p_{2}\right)$ after the spraying of pesticides every time for model (2). To take account of the resource limitation and saturation effects, we use the Hill function [31], and then model (2) can be rewritten as

$$
\begin{aligned}
\dot{x} & =a x-b x^{2}-\frac{c x y}{1+\omega x}, \quad t \neq n T, n \in \mathcal{N}, \\
\dot{y} & =\mu \frac{c x y}{1+\omega x}-d y, \\
x\left(t^{+}\right) & =\left(1-\frac{p_{1}^{\max } x(t)}{x(t)+\theta_{1}}\right) x(t), \\
y\left(t^{+}\right)= & \left(1-\frac{p_{2}^{\max } y(t)}{y(t)+\theta_{2}}\right) y(t)+\sigma,
\end{aligned}
$$

with nonnegative initial value $\left(x_{0}, y_{0}\right)$, and $p_{1}^{\max }\left(p_{2}^{\max }\right)$ and $\theta_{1}\left(\theta_{2}\right)$ represent the maximal fatality rate and the half saturation constant for pest (natural enemy), respectively. That is, we can use a combination of biological (periodic releasing of natural enemies) and chemical (spraying pesticide) tactics to suppress the pest to a low level. 


\section{Mathematical Analysis of the Pest-Free Periodic Solution and Threshold Conditions}

In this section, we first demonstrate the existence of the pestfree periodic solution of model (3) and focus on determining its global attractivity. Moreover, threshold values are derived, and LHS/PRCCs analysis techniques are employed to investigate the key control parameters which are most significantly related to threshold values.

3.1. Existence of the Pest-Free Periodic Solution. In order to illustrate the existence of the pest-free periodic solution, we first focus on the pest-free set: $\left\{(x, y) \in \mathscr{R}_{+}^{2}, x=0\right\}$, which is clearly invariant by model (3); within this set, model (3) becomes

$$
\begin{gathered}
\dot{y}=-d y(t), \quad t \neq n T, \\
y\left(t^{+}\right)=\left(1-\frac{p_{2}^{\max } y(t)}{y(t)+\theta_{2}}\right) y(t)+\sigma, \quad t=n T, \\
y\left(0^{+}\right)=y_{0} .
\end{gathered}
$$

Solving the above equation in interval $(n T,(n+1) T]$, one yields

$$
\begin{aligned}
y & \left((n+1) T^{+}\right) \\
& =\left(1-\frac{p_{2}^{\max } y\left(n T^{+}\right) \mathrm{e}^{-d T}}{y\left(n T^{+}\right) \mathrm{e}^{-d T}+\theta_{2}}\right) y\left(n T^{+}\right) \mathrm{e}^{-d T}+\sigma .
\end{aligned}
$$

Denote $y\left(n T^{+}\right)=y_{n}$; then the above equation can be rewritten as the following difference equation:

$$
\begin{aligned}
y_{n+1} & =\frac{\left(1-p_{2}^{\max }\right) \mathrm{e}^{-2 d T} y_{n}^{2}+\theta_{2} \mathrm{e}^{-d T} y_{n}}{\mathrm{e}^{-d T} y_{n}+\theta_{2}}+\sigma \\
& \doteq F\left(y_{n}\right),
\end{aligned}
$$

which is the so-called stroboscopic map of model (4), and describes the relations of the number of natural enemies between any two successive pulse points. Consequently, the existence of the positive steady state of model (6) implies the existence of a positive periodic solution of model (4). Therefore, we first discuss the existence of a positive steady state of (6); that is, the derivative of $F\left(y_{n}\right)$ with respect to $y_{n}$ yields

$$
F^{\prime}\left(y_{n}\right)=\left[\left(1-p_{2}^{\max }\right)+\frac{p_{2}^{\max } \theta_{2}^{2}}{\left(\mathrm{e}^{-d T} y_{n}+\theta_{2}\right)^{2}}\right] \mathrm{e}^{-d T}
$$

and it is easy to see that $0<F^{\prime}\left(y_{n}\right)<1$ holds true for all $y_{n}$.

Next, we discuss the positive fixed point of the stroboscopic map (6), denoted by $y^{*}$, which satisfies the following equation:

$$
A_{1} y^{* 2}+B_{1} y^{*}+C_{1}=0
$$

where $A_{1}=\exp (-d T)\left[1-\left(1-p_{2}^{\max }\right) \exp (-d T)\right]>0, B_{1}=$ $\theta_{2}-\sigma \exp (-d T)-\theta_{2} \exp (-d T), C_{1}=-\theta_{2} \sigma<0$.
Denote $\Delta=B_{1}^{2}-4 A_{1} C_{1}$, and by simple calculation, we have

$$
\begin{aligned}
\Delta= & \left(\theta_{2}-\sigma \mathrm{e}^{-d T}-\theta_{2} \mathrm{e}^{-d T}\right)^{2} \\
& +4 \theta_{2} \sigma \mathrm{e}^{-d T}\left[1-\left(1-p_{2}^{\max }\right) \mathrm{e}^{-d T}\right]>0 .
\end{aligned}
$$

Obviously, (6) has a unique positive root; that is,

$$
y^{*}=\frac{-B_{1}+\sqrt{B_{1}^{2}-4 A_{1} C_{1}}}{2 A_{1}},
$$

which is stable due to $0<F^{\prime}\left(y^{*}\right)<1$.

According to the relations between a fixed point of the stroboscopic map (6) and the periodic solution of model (4), we conclude that the submodel (4) of (3) has a unique nontrivial positive periodic solution, denoted by $y_{p}(t)$ and

$$
y_{p}(t)=y^{*} \exp [-d(t-n T)] \quad \text { for } n T<t \leq(n+1) T \text {, }
$$

and it follows from $0<F^{\prime}\left(y_{n}\right)<1$ and [32] that we have the following theorem.

Theorem 1. Model (4) has a positive periodic solution $y_{p}(t)$ with periodic $T$ and for every solution $y(t)$ of (4) such that $\left|y(t)-y_{p}(t)\right| \rightarrow 0$ as $t \rightarrow+\infty$, where $y_{p}(t)=y^{*} \mathrm{e}^{-d(t-n T)}$ and $y^{*}$ is defined in (10).

By using Theorem 1, we conclude that the unique fixed point $y^{*}$ of (6) is globally stable. Accordingly, the stability of the positive periodic solution is determined by the stability of the positive equilibria of the difference equation made by the sequence of impulsive points. So, the positive periodic solution $y_{p}(t)$ of model (4) is globally stable.

Therefore, we obtain the general expression of an unique pest-free periodic solution of model (3) over the interval $n T<$ $t \leq(n+1) T$ for all $n \in \mathcal{N}$ denoted by

$$
\left(x_{p}(t), y_{p}(t)\right)=\left(0, y^{*} \mathrm{e}^{-d(t-n T)}\right) \text {. }
$$

3.2. Stability of the Pest-Free Periodic Solution. The threshold conditions which guarantee the global stability of the pestfree periodic solution (12) play a key role in pest control. To show this, we now concentrate on the stability of the pest-free solution (12) for model (3). We change model (3) variables to consider the deviations from the pest-free solution that are denoted by $(\bar{x}(t), \bar{y}(t))$ so that

$$
(\bar{x}(t), \bar{y}(t))=(x(t), y(t))-\left(x_{p}(t), y_{p}(t)\right)
$$

that yields

$$
\begin{gathered}
\dot{\bar{x}}=a \bar{x}-b \bar{x}^{2}-\frac{c \bar{x}}{1+\omega \bar{x}}\left[\bar{y}+y_{p}(t)\right], \\
\dot{\bar{y}}=\frac{\mu c \bar{x}}{1+\omega \bar{x}}\left[\bar{y}+y_{p}(t)\right]-d \bar{y} .
\end{gathered}
$$


Assuming that $(\bar{x}, \bar{y})$ are small enough, we get the linear approximation of the deviation system around the periodic solution $\left(x_{p}(t), y_{p}(t)\right)$,

$$
\begin{aligned}
& \dot{\bar{x}}=\left(a-c y_{p}(t)\right) \bar{x}, \\
& \dot{\bar{y}}=\mu c y_{p}(t) \bar{x}-d \bar{y} .
\end{aligned}
$$
set

Before stating Theorem 2, for the sake of convenience, we

$$
\begin{gathered}
R_{0}^{1}=\frac{a d T}{c y^{*}\left(1-\mathrm{e}^{-d T}\right)}, \\
R_{0}^{2}=\frac{c d T M_{s}}{c y^{*}\left(1-\mathrm{e}^{-d T}\right)-d \ln \left(1-p_{1}^{\max }\right)},
\end{gathered}
$$

where $M_{s}=(a \omega+b)^{2} /(4 b c \omega)$.

We are now in a position to present the sufficient conditions for the global stability of the pest-free periodic solution $\left(x_{p}(t), y_{p}(t)\right)$ of model (3) as follows.

Theorem 2. The pest-free periodic solution $\left(x_{p}(t), y_{p}(t)\right)$ of model (3) is locally stable in the first quadrant, provided that $R_{0}^{1} \leq 1$ and is globally stable if $\max \left\{R_{0}^{1}, R_{0}^{2}\right\} \leq 1$.

The proof of Theorem 2 is given in Appendix A.

Remark 3. According to the formulae $R_{0}^{1}$ and $R_{0}^{2}$, we have

$$
\begin{aligned}
R_{0}^{1}- & R_{0}^{2} \\
& =\frac{d T\left[y^{*}\left(1-\mathrm{e}^{-d T}\right)\left(a^{2}-c^{2} M_{s}\right)-a d \ln \left(1-p_{1}^{\max }\right)\right]}{c y^{*}\left(1-\mathrm{e}^{-d T}\right)\left[a y^{*}\left(1-\mathrm{e}^{-d T}\right)-d \ln \left(1-p_{1}^{\max }\right)\right]} .
\end{aligned}
$$

Therefore, if $y^{*}\left(1-\mathrm{e}^{-d T}\right)\left(a^{2}-c^{2} M_{s}\right)-a d \ln \left(1-p_{1}^{\max }\right)>$ 0 , then $R_{0}^{1}>R_{0}^{2}$, and consequently the pest-free periodic solution $\left(x_{p}(t), y_{p}(t)\right)$ of model (3) is globally stable if $R_{0}^{1}<$ 1 , which shows that the local stability indicates the global stability. If $y^{*}\left(1-\mathrm{e}^{-d T}\right)\left(a^{2}-c^{2} M_{s}\right)-a d \ln \left(1-p_{1}^{\max }\right)<0$, then $R_{0}^{1}<R_{0}^{2}$ (see Figure 1(a)). Thus, the pest-free periodic solution $\left(x_{p}(t), y_{p}(t)\right)$ of model (3) is globally stable if $R_{0}^{2}<1$ (see Figure 1(b)). If so, we cannot show that in this case the local stability indicates the global stability theoretically.

3.3. Threshold Conditions. Note that although the threshold values $R_{0}^{i}$ depend on all parameters of model (3), the most interesting parameters here are the pulse period $T$ and some parameters related to resource limitation such as the maximum fatality rates $p_{i}^{\max }(i=1,2)$ and the half saturation constant $\theta_{2}$, so it makes sense to know how $T, p_{i}^{\max }, \theta_{2}$ affect the threshold conditions $\left(R_{0}^{i}<1 ; i=1,2\right)$ which guarantee the global stability of the pest-free periodic solution (12). Figure 1(a) indicates that $R_{0}^{i}(i=1,2)$ are monotonic increasing functions with respect to $T \in(0,20]$. Moreover, there are some critical values of $T$ such that $R_{0}^{i}=1$, which indicate that there exists one maximum allowable impulsive period $T_{i}^{\max }$ such that $R_{0}^{i}<1$ for all $T<T_{i}^{\max }$ and $i=1,2$, respectively. Meanwhile, $R_{0}^{2}$ takes more time to reach the threshold value 1 than $R_{0}^{1}$ as a result of $T_{1}^{\max }<T_{2}^{\max }$. In more detail, the contour plots of Figures 2(a) and 2(c) show that the control strategies (decreasing $p_{2}^{\max }$, increasing $\theta_{2}$ ) can reduce the threshold value $R_{0}^{i}(i=1,2)$. However, increasing $p_{1}^{\max }$ can reduce the threshold value $R_{0}^{2}$ from Figure $2(\mathrm{~d})$. Therefore, in order to control the pest population, it is very important to increase the maximum fatality rate $p_{1}^{\max }$ for the pest while decreasing the maximum fatality rate $p_{2}^{\max }$ for the natural enemy, which means that it is essential to increase the pesticide's efficacy and reduce its effectiveness against the natural enemy while enhancing the efficiency of the natural enemies. Also, it is important to carry out pulsed control actions more and more frequently under resource limitation according to Figure 2(b), and then we can eradicate the pest successfully.

3.4. Sensitivity to Variations. Previous analysis indicates that $R_{0}^{i}(i=1,2)$ are significant threshold conditions affecting outbreaks of the pest population. Sensitivity analysis of the most significant parameters (such as intrinsic growth rate $a$, death rate $c$, the maximum fatality rate $p_{i}^{\max }$, half saturation constant $\theta_{2}$, constant stocking number $\sigma$, and pulse period $T$ ) was performed by evaluating the PRCCs for various input parameters against the threshold condition $R_{0}^{i}(i=1,2)$ with the LHS method (see $[24,25,33]$ ). PRCCs show how influential each parameter is on $R_{0}^{i}(i=1,2)$. Figure 3 shows the PRCCs results which illustrate the dependence of $R_{0}^{i}(i=1,2)$ on each parameter, and we considered $|\mathrm{PRCC}| \epsilon$ $(0.4, \infty),(0.2,0.4),(0,0.2)$ as indicating correlations of very important, moderate, or little significance between input parameters and output variables, respectively. The positive sign of their PRCCs indicates that if the parameters are increased, the value of $R_{0}^{2}$ increases and vice versa for negative signs.

By using LHS with 3,000 samples, uncertainty and sensitivity analyses for all parameters in model (3) were determined. A uniform distribution function was used and tested for significant PRCCs for all parameters with wide ranges, making use of the data from Table 1, and the baseline values of all parameters are given in the figure legend of Figure 3. The PRCCs sensitivity analysis on $R_{0}^{1}$ and $R_{0}^{2}$ between all parameters is depicted in Figures 3(a) and 3(b), which shows that variations in the intrinsic growth rate $a$, the death rate $c$, the pulse period $T$, and the constant stocking rate $\sigma$ have the four greatest effects on the outbreak of the pest with the first three increasing the $R_{0}^{i}(i=1,2)$ and the latter decreasing them. The half saturation constant $\theta_{2}$ generates minor decreasing impact on two thresholds with similar amounts of influence in both figures. The maximum fatality rate $p_{2}^{\max }$ has a minor increasing effect on $R_{0}^{i}(i=1,2)$. However, the other maximum fatality rate $p_{1}^{\max }$ has a great decreasing impact on $R_{0}^{2}$; that is, $p_{1}^{\max }$ plays an important role in the global stability, while it is without influence upon the local stability of $\left(x_{p}(t), y_{p}(t)\right)$ of model (3). 


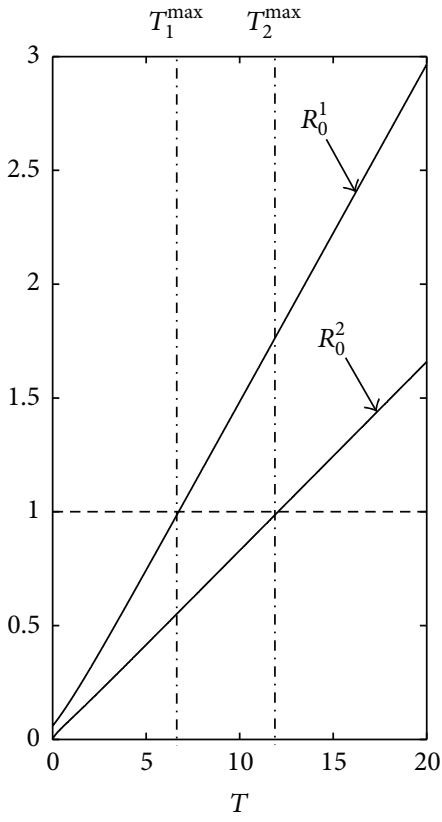

(a)

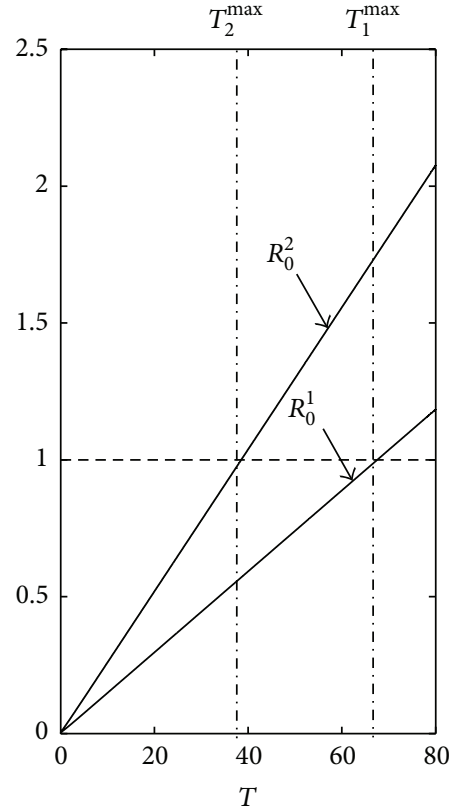

(b)

FIgURE 1: The monotonicity of $R_{0}^{1}$ and $R_{0}^{2}$ with respect to $T$. (a) $R_{0}^{1}>R_{2}$. All parameters are fixed as follows: $a=0.5, b=0.8, c=1.6, \omega=1$, $\mu=0.4, d=0.37, \sigma=0.78, p_{1}^{\max }=0.95, p_{2}^{\max }=0.2, \theta_{1}=1, \theta_{2}=2$. (b) $R_{0}^{1}<R_{2}$. All parameters are fixed as follows: $a=0.1, b=0.8, c=2.5$, $\omega=1, \mu=0.4, d=0.37, \sigma=1, p_{1}^{\max }=0.95, p_{2}^{\max }=0.1, \theta_{1}=1$, and $\theta_{2}=2$.

TABLE 1: The main coefficient values of model (3). All other parameters are fixed as follows: $b=0.5, c=1.6, \omega=0.9$, and $\mu=0.4$.

\begin{tabular}{lccccccc}
\hline Parameter & $a$ & $d$ & $p_{1}^{\max }$ & $p_{2}^{\max }$ & $\theta_{2}$ & $\sigma$ \\
\hline Definition & $\begin{array}{c}\text { Intrinsic } \\
\text { growth rate }\end{array}$ & Death rate & $\begin{array}{c}\text { Maximum } \\
\text { fatality rate }\end{array}$ & $\begin{array}{c}\text { Maximum } \\
\text { fatality rate }\end{array}$ & $\begin{array}{c}\text { Half saturation } \\
\text { constant }\end{array}$ & $\begin{array}{c}\text { Constant } \\
\text { stocking } \\
\text { number }\end{array}$ & $\begin{array}{c}\text { Pulse period } \\
\text { Range }\end{array}$ \\
\hline
\end{tabular}

So far, the dynamics of the pest-free periodic solution (12) of model (3) with limited resource has been investigated completely. The results demonstrate that the pest population can be eradicated, provided that certain conditions are satisfied.

\section{The Existence of the Positive Periodic Solution}

In this section, we adopt bifurcation theory [34] to analyze the existence of a positive periodic solution of model (3) near the pest-free periodic solution by setting the impulsive period $T$ and constant stocking number $\sigma$ as bifurcation parameters. According to the bifurcation theorem in [34], we first obtain the following result.

Theorem 4. The supercritical branch occurs at the point $T_{0}>$ 0 satisfying $R_{0}^{1}\left(T_{0}\right)=1$; that is, it will have a stable positive periodic solution when $T>T_{0}$ and is close to $T_{0}$, provided one of the following conditions of model (3) holds

$\left(\mathrm{C}_{1}\right) M_{1}<0$ and $1-A_{3} A_{4}<0$;

$\left(\mathrm{C}_{2}\right) M_{1}>0$ and $N_{1}+N_{2}<0$, where

$$
\begin{aligned}
& M_{1}=-c d T_{0} y^{*}\left(T_{0}\right) \cdot \frac{A_{3} \mathrm{e}^{-d T_{0}}}{1-A_{3} \mathrm{e}^{-d T_{0}}}-\left[a-c y_{p}\left(T_{0}\right)\right] ; \\
& N_{1}=2 p_{1}^{\max } \theta_{1}^{-1} \cdot \mathrm{e}^{2 \int_{0}^{T_{0}}\left[a-c y_{p}(\xi)\right] \mathrm{d} \xi}-A_{5} ; \\
& N_{2}=-2 c T_{0} \cdot \frac{1-A_{3} A_{4}}{1-A_{3} \mathrm{e}^{-d T_{0}}} \cdot \mathrm{e}^{\int_{0}^{T_{0}}\left[a-c y_{p}(\xi)\right] \mathrm{d} \xi} ; \\
& A_{3}=1-p_{2}^{\max }+\frac{p_{2}^{\max } \theta_{2}^{2}}{\left[y_{p}\left(T_{0}\right)+\theta_{2}\right]^{2}} ; \\
& A_{4}=\mu c \int_{0}^{T_{0}} y_{p}(v) \cdot \mathrm{e}^{-d\left(T_{0}-v\right)} \cdot \mathrm{e}^{\int_{0}^{v}\left[a-c y_{p}(\xi)\right] \mathrm{d} \xi} \mathrm{d} v ; \\
& A_{5}=-2 T_{0} \cdot\left[b+c \omega y_{p}(v)\right] \cdot \mathrm{e}^{\int_{0}^{T_{0}}\left[a-c y_{p}(\xi)\right] \mathrm{d} \xi} \\
& -c \int_{0}^{T_{0}}\left\{e^{T_{v}^{T_{0}}\left[a-c y_{p}(\xi)\right] \mathrm{d} \xi}\right.
\end{aligned}
$$




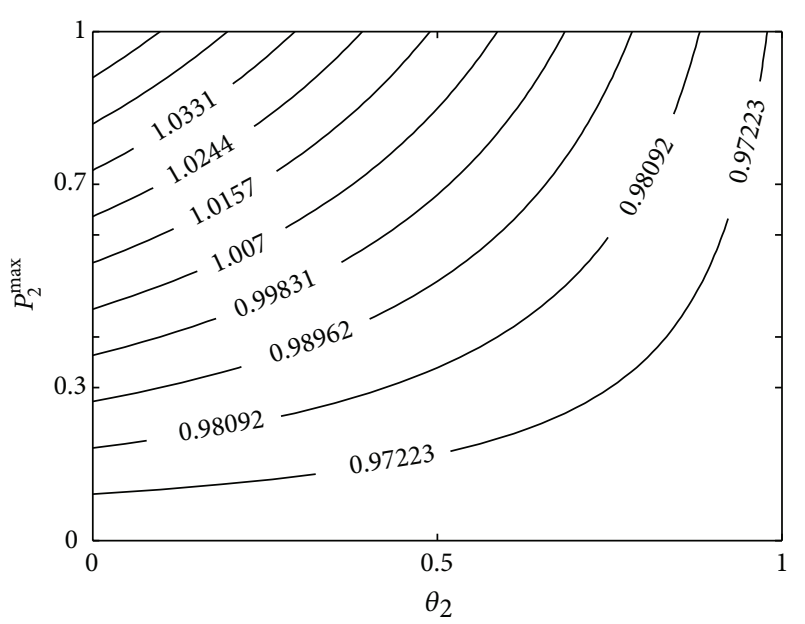

(a)

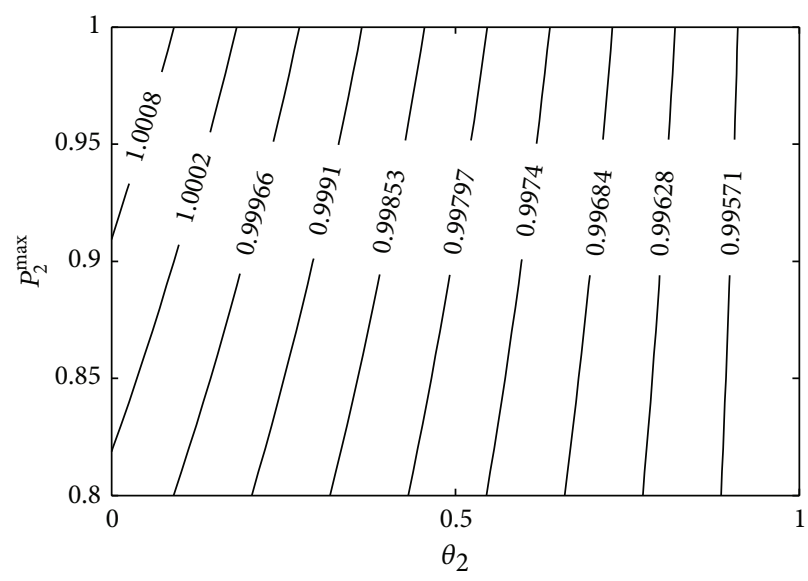

(c)

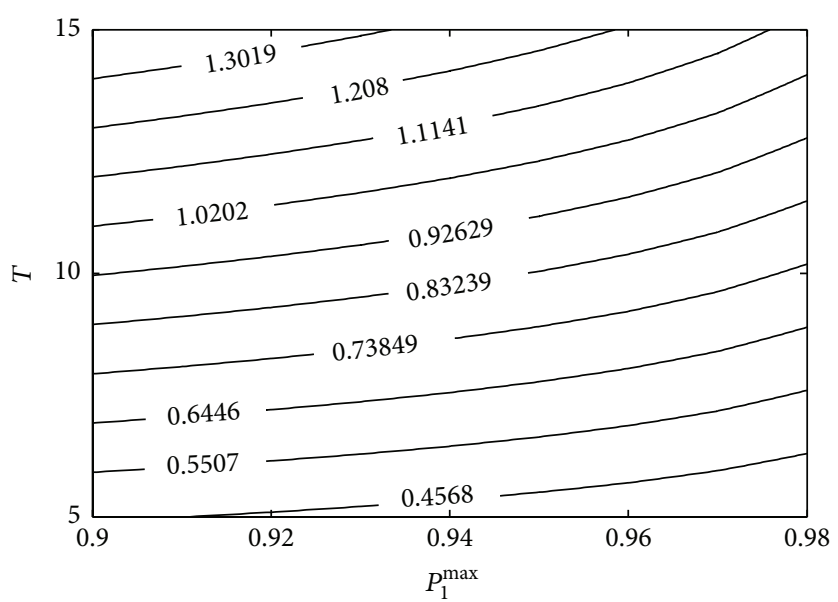

(b)

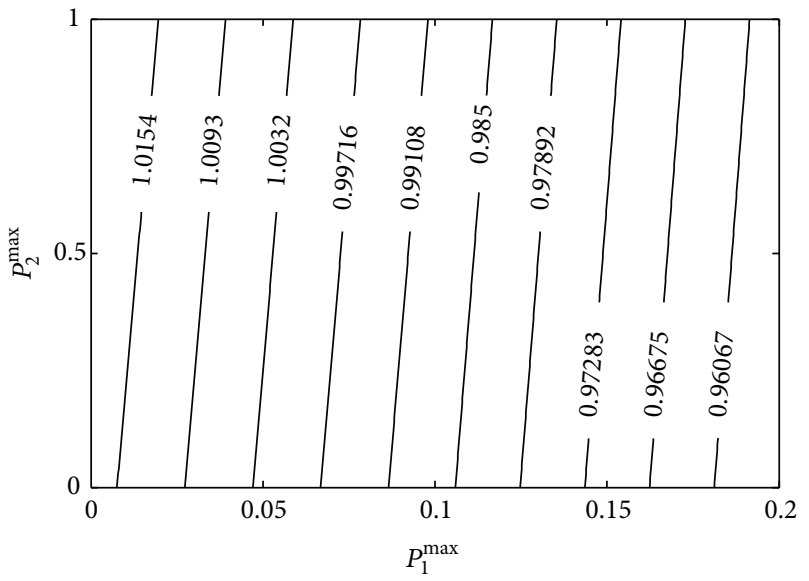

(d)

FIgURE 2: Contour plots of $R_{0}^{1}$ and $R_{0}^{2}$ : (a) plot of contours of $R_{0}^{1}$ versus $p_{2}^{\max }$ and $\theta_{2}$; (b) plot of contours of $R_{0}^{2}$ versus $T$ and $p_{1}^{\max }$; (c) plot of contours of $R_{0}^{2}$ versus $p_{2}^{\max }$ and $\theta_{2}$; (d) plot of contours of $R_{0}^{1}$ versus $p_{2}^{\max }$ and $p_{1}^{\max }$. All other parameters are fixed as follows: $a=0.5, b=0.8$, $c=1.6, \omega=1, \mu=0.4, d=0.37, \sigma=0.78, p_{1}^{\max }=0.95, p_{2}^{\max }=0.2, \theta_{1}=1, \theta_{2}=2, T=6.5(A)$, and $T=12(C-D)$.

$$
\begin{aligned}
& \int_{0}^{v}\left\{\mu c y_{p}(\theta) \cdot \mathrm{e}^{-d(v-\theta)}\right. \\
&\left.\left.\cdot \mathrm{e}^{\int_{0}^{\theta}\left[a-c y_{p}(\xi)\right] \mathrm{d} \xi}\right\} \mathrm{d} \theta\right\} \mathrm{d} v .
\end{aligned}
$$

The proof of Theorem 4 is given in Appendix B. Theorem 4 shows that there exists a positive periodic solution under some conditions, providing that the pestfree periodic solution becomes unstable. It follows from Theorem 4 that if $T>T_{0}$ and is closed to $T_{0}$, the periodic solution of model (3) is stable.

Figure 4(a) indicated that if the pulse period $T$ is more than $T_{1}^{\max }$, the pest-free solution becomes unstable and variable $x$ begins to oscillate with a large amplitude that corresponds to periodic outbreaks of the pest population. If the pulse period is further increased, a sequence of period adding bifurcations interchanging with regions of chaos is observed. Figures 4 and 5 show that model (3) has more complex and interesting dynamic behaviors including periodic doubling bifurcation, chaotic solutions, quasiperiodic solutions, tangent bifurcation, multistability, chaos crisis, periodic windows, periodic halving bifurcation, and crises with the increasing of pulse period $T$ and constant stocking number $\sigma$, respectively. For example, Figures 4(a) and 4(b) show that for model (3) there exists a quasiperiodic solution when $T=8$; see Figure 6 for full details.

Meanwhile, bifurcation analyses also indicate that multiple attractors can coexist for a wide range of parameters, for example, the two attractors with quite different pest amplitudes that can coexist when $\sigma=7.3$ in Figure 5; see Figure 7 for more details.

Furthermore, we can clearly see that there are two examples of attractor coexistence of model (3) when $\sigma=$ 4.37 in Figure 5; when small changes are introduced in initial value, numerical simulations imply that this solution can switch to other attractors, that is, the switch-like transitions between the two attractors shown in Figure 8 with $\left(x_{0}, y_{0}\right)=$ $(2,5)$. 


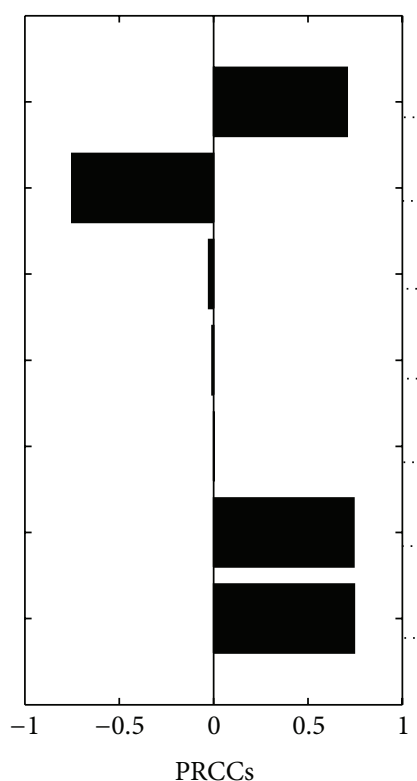

(a)

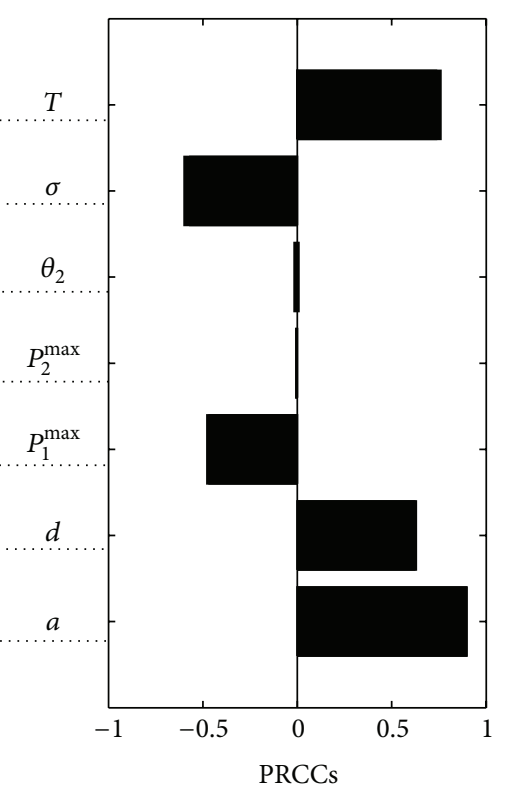

(b)

FIGURE 3: Sensitivity analysis of thresholds $R_{0}^{1}$ and $R_{0}^{2}$ : the baseline parameter values are $a=0.8, b=0.5, c=1.6, \omega=0.9, \mu=0.4, d=0.3$, $\sigma=0.5, p_{1}^{\max }=0.95, p_{2}^{\max }=0.2, \theta_{1}=0.7, \theta_{2}=2$, and $T=1$. (a) PRCC results with $R_{0}^{1}$; (b) PRCC results with $R_{0}^{2}$.

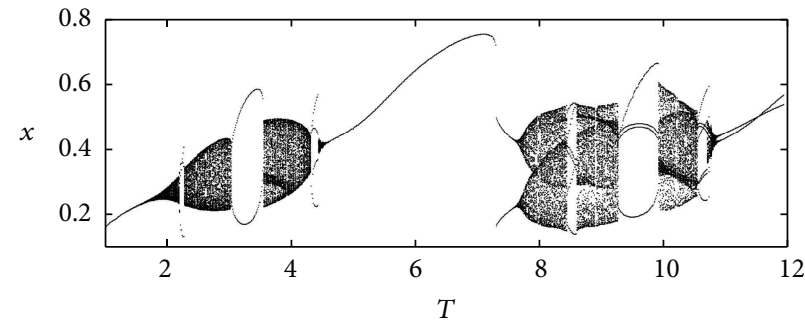

(a)

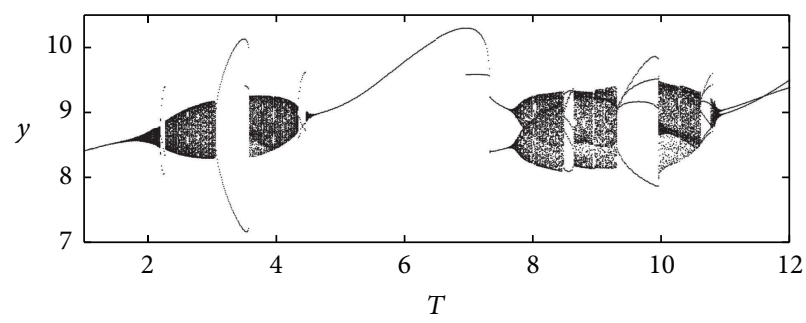

(b)

FIGURE 4: Bifurcation diagrams of model (3) with respect to bifurcation parameter $T$, where all parameter values are fixed as follows: $a=8$, $b=5, c=1, \omega=1, \mu=0.95, d=0.2, \sigma=0.8, p_{1}^{\max }=0.5, p_{2}^{\max }=0.01, \theta_{1}=0.2, \theta_{2}=2$, and $\left(x_{0}, y_{0}\right)=(2,1)$. (a) Prey population $x$ with $T$; (b) predator population $y$ with $T$.

In summary, numerical simulations show that with resource limitation model (3) has more and more complex dynamics. It is well known that the dosages of pesticide applied and numbers of natural enemies released are crucial for controlling pests, but when these key factors of controlling pests are limited, it makes it more difficult for pest control.

\section{Discussion and Biological Conclusions}

The main purpose of this paper was to understand the effect of resource limitation on outbreaks of a pest population, so we conducted a dynamical analysis of a Holling II predator-prey model which incorporates a nonlinear pulse. To this end, we introduced a nonlinear form $p_{i}(\phi)=p_{i}^{\max } \phi /\left(\phi+\theta_{i}\right)(i=1$, $\phi=x$; or $i=2, \phi=y$ ) as fatality rate for pest or natural enemy. The nonlinear fatality rate taking density dependence into consideration gives a better portrayal of the reality, which can be used to obtain a more accurate evaluation of the pestnatural enemy system. However, it is worth noting that the mathematical model described here differs from the ones discussed by many other researchers since we have taken the nonlinear impulsive function into account. The nonlinear nature of the impulsive functions makes the dynamical behavior of solutions change dramatically and it gets more and more complicated, which means that it is a very difficult task to control pests under resource limitation. We discuss the control strategy for the pest through theoretical analysis and numerical simulations.

The results indicate that pesticides and natural enemies as two approaches to control pests must be committed to a long-term strategy especially when being under resource limitation. Stability analysis for model (3) shows that $R_{0}^{i}$ could be a global threshold in a sense that if all these control methods are adopted so that $R_{0}^{i}<1 \quad(i=1,2)$, then the strategies are effective enough to eradicate the pest population. We adopt LHS/PRCCs uncertainty and sensitivity analysis techniques to gain a better understanding of the impacts of 


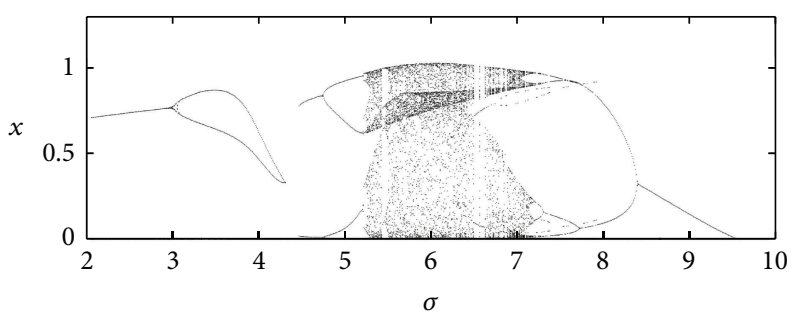

(a)

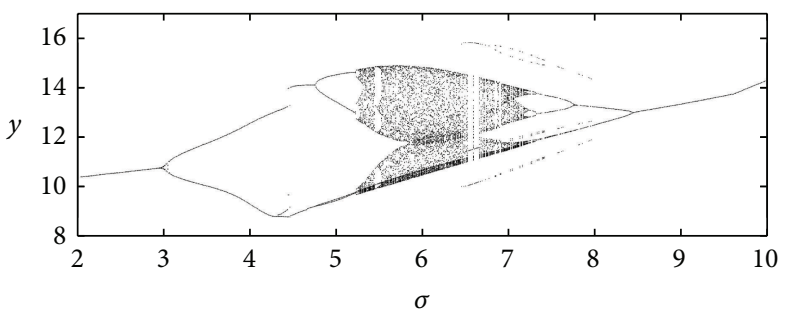

(b)

FIGURE 5: Bifurcation diagrams of model (3) with respect to bifurcation parameter $\sigma$. (a) Prey population $x$ with $\sigma$; (b) predator population $y$ with $\sigma$. The other parameters are identical to those in Figure 4 and $T=0.5$.

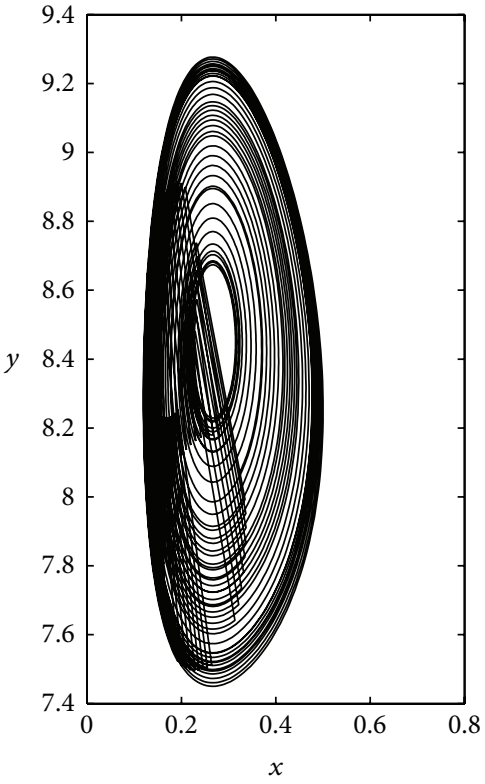

(a)

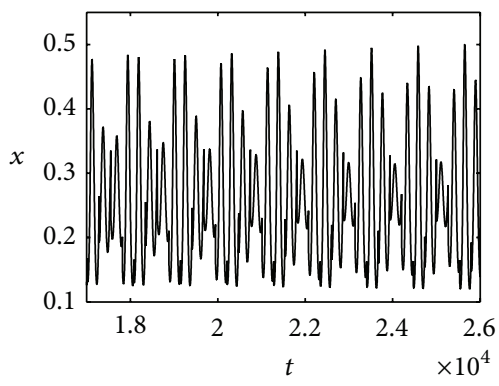

(b)

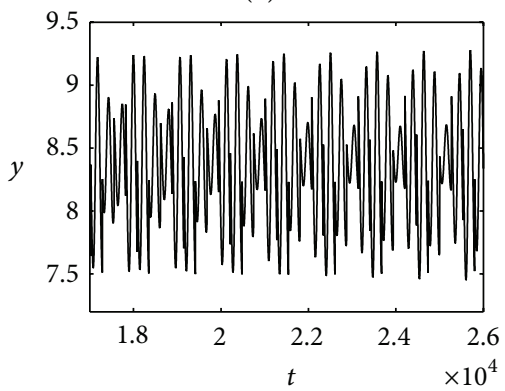

(c)

Figure 6: A typical quasiperiodic solution of model (3) over $t \in[17000,26000]$. (a) Phase portrait of $x$ and $y$ with $t$; (b) time series of $x$; (c) time series of $y$. The other parameters are identical to those in Figure 4 and $T=8$.

parameters on the threshold when various parameters are changed within the ranges of values observed empirically (see Table 1 and Figure 3). The results illustrate that we should pay more attention to the impulsive period $T$, maximum fatality rate $p_{1}^{\max }$ for the pest, and constant stocking number $\sigma$ which maximizes the threshold values $R_{0}^{i}$ to prevent outbreaks of the pest population. That is, it is essential to increase the pesticide efficacy and the efficiency of the natural enemy. Furthermore, when resources are limited, it is important to carry out pesticide and natural enemy programs more frequently than when resources are not limited. Our results also confirm that IPM is the optimal control strategy.

Based on Theorem 4, the results demonstrate that the dynamic behavior of model (3) may be dramatically affected by small changes in the value of initial densities of pest and natural enemy with resource limitation. By choosing impulsive perturbations $\sigma$ and $T$ as bifurcation parameters, we have obtained bifurcation diagrams (see Figures 4 and 5) including periodic doubling bifurcation, chaotic solutions, quasiperiodic solutions (see Figure 6 for details), tangent bifurcation, multistability (see Figure 7 for details), chaos crisis, periodic windows, periodic halving bifurcation, and crises, which can help us to further understand the application of nonlinear pulses in our model (for more information about bifurcation diagrams, refer to $[15,18,35])$. According to bifurcation diagrams, we can find that the routes to chaos are very complicated; that is, with resource limitation, there are several hidden factors that can adversely affect our control strategy. The increasing number of potential complexities predicted by the theory does not seem to make this task any easier. Nevertheless, identifying complicated, possibly chaotic, dynamics in IPM models may present a major challenge for controlling the pest populations in practice.

In our study, we have investigated the dynamical behavior of a Holling II predator-prey model to understand how the limited resource affects pest outbreaks. To link the costs of developing and implementing controls to population dynamic modeling of disease epidemics, it is necessary to 


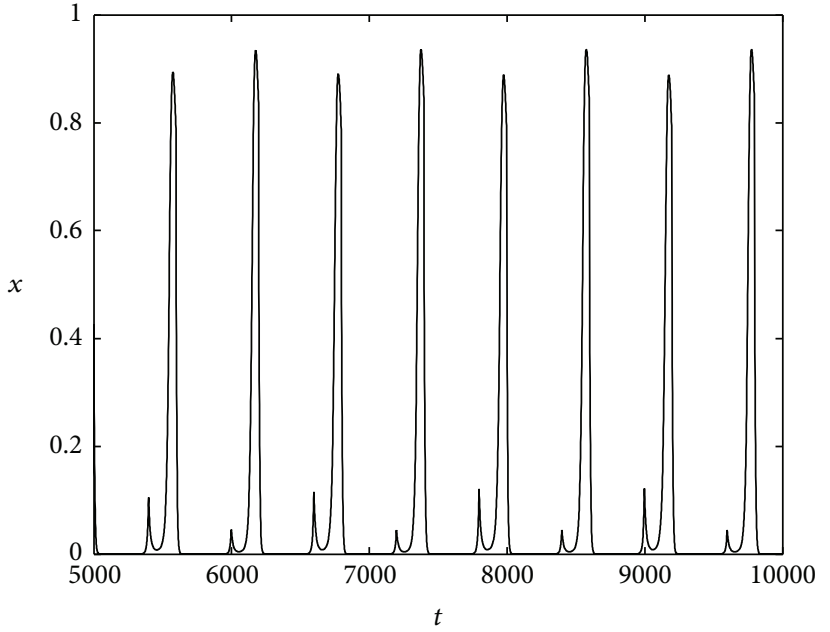

(a)

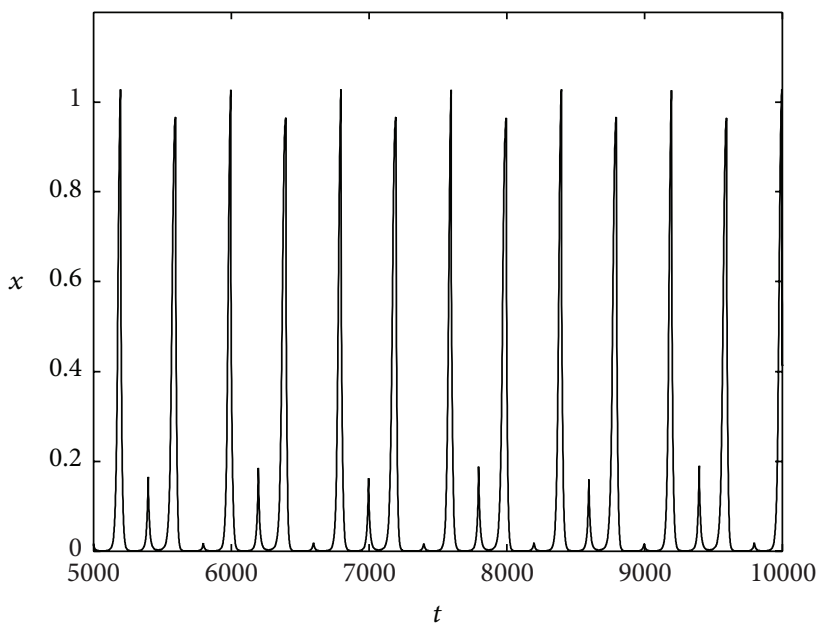

(c)

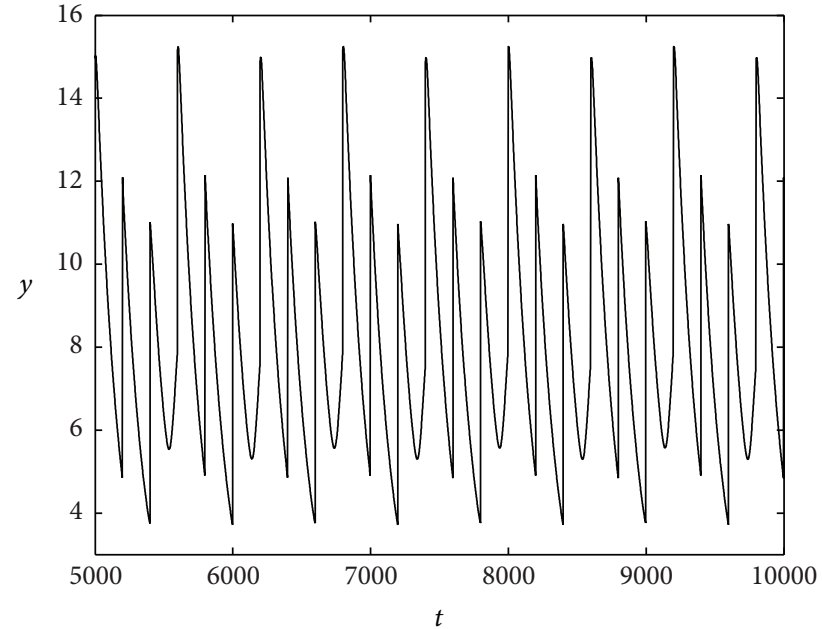

(b)

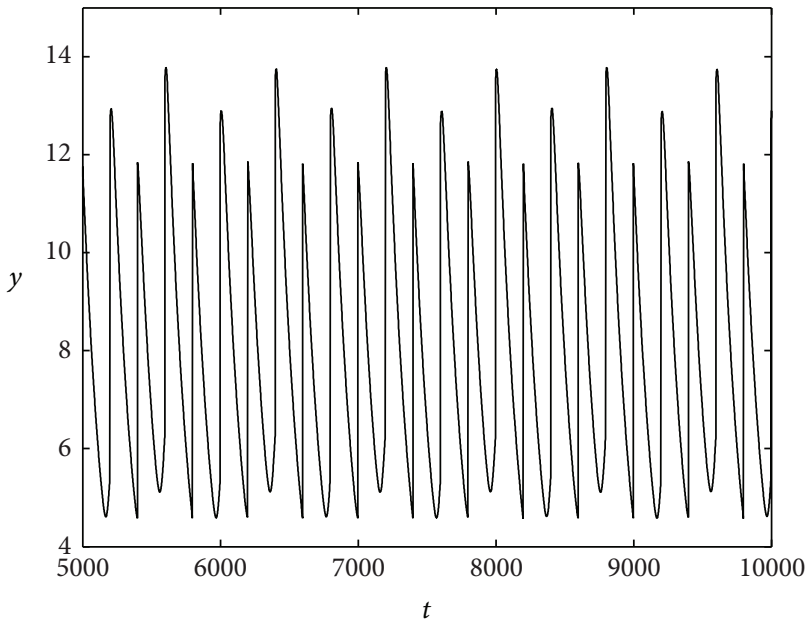

(d)

Figure 7: Two coexisting attractors of model (3) with $\sigma=7.3$. The initial values from left to right are $\left(x_{0}, y_{0}\right)=(2.5,1),(2,5.1)$, respectively. (a-b) Periodic attractor with period 12; (c-d) periodic attractor with period 16. The other parameters are identical to those in Figure 4 and $T=6$.

consider other resource limitation factors such as delayed responses and residual effects on pests of pesticides, as such nonlinear factors can affect the success of pest control. We leave these topics for further investigations.

\section{Appendices}

\section{A. The Proof of Theorem 2}

To prove the local stability of the solution (12) of model (3), we need to investigate the difference equation determined by the impulsive periodic $T$ with respect to the linear equation (15). Let $\Phi(T)$ be the fundamental matrix of (15); thus $\Phi(T)$ must satisfy

$$
\Phi(T)=\left(\begin{array}{cc}
\mathrm{e}_{0}^{T}\left[a-c y_{p}(t)\right] \mathrm{d} t & 0 \\
\star & \mathrm{e}^{-d T}
\end{array}\right)
$$

where $\Phi(0)=I$ is the identity matrix and the term $\star$ is not necessarily computed in detail as it is not required in the following analysis.

Resetting the impulsive conditions of model (3) from the last two equations gives

$$
\begin{aligned}
\left(\begin{array}{l}
\bar{x}\left(n T^{+}\right) \\
\bar{y}\left(n T^{+}\right)
\end{array}\right)= & \left(\begin{array}{cc}
1 & 0 \\
0 & 1-p_{2}^{\max }+\frac{p_{2}^{\max } \theta_{2}^{2}}{\theta_{2}+y_{p}(n T)}
\end{array}\right) \\
& \times\left(\begin{array}{l}
\bar{x}(n T) \\
\bar{y}(n T)
\end{array}\right)=B(n T)\left(\begin{array}{l}
\bar{x}\left(n T^{+}\right) \\
\bar{y}\left(n T^{+}\right)
\end{array}\right) .
\end{aligned}
$$

Then, two Floquet multiplies of the matrix

$$
M=B(T) \Phi(T)
$$




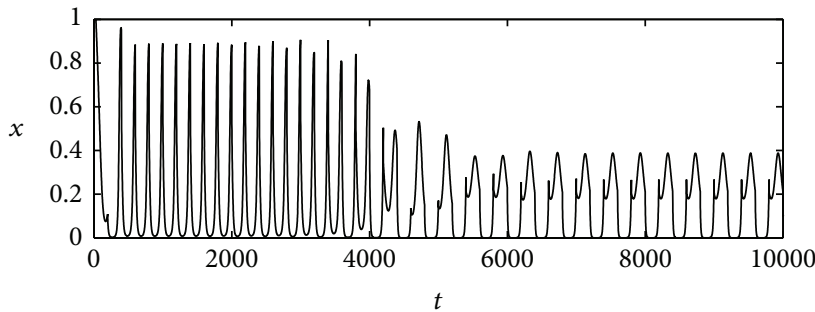

(a)

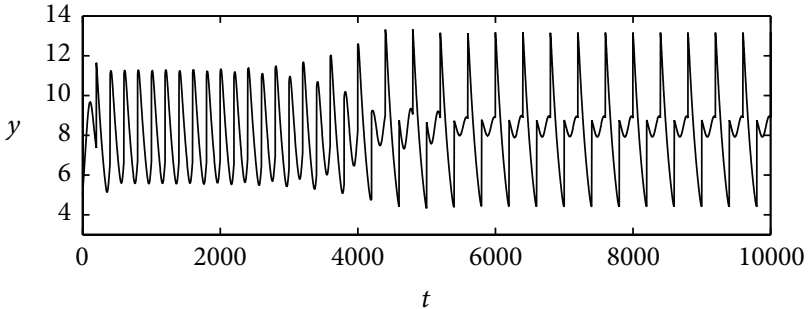

(b)

Figure 8: Attractors switch-like behavior of model (3) with $\sigma=4.37$ and $\left(x_{0}, y_{0}\right)=(2,5)$. (a) Time series of $x$; (b) time series of $y$. The other parameters are identical to those in Figure 7.

$$
\begin{aligned}
= & \left(\begin{array}{cc}
1 & 0 \\
0 & 1-p_{2}^{\max }+\frac{p_{2}^{\max } \theta_{2}^{2}}{\theta_{2}+y_{p}(T)}
\end{array}\right) \\
& \times\left(\begin{array}{cc}
\mathrm{e}^{\int_{0}^{T}\left[a-c y_{p}(t)\right] \mathrm{d} t} & 0 \\
\star & \mathrm{e}^{-d T}
\end{array}\right)
\end{aligned}
$$

are $\lambda_{1}=\exp \left\{\int_{0}^{T}\left[a-c y_{p}(t)\right] \mathrm{d} t\right\}, \lambda_{2}=\left\{1-p_{2}^{\max }+p_{2}^{\max } \theta_{2}^{2} /\left[\theta_{2}+\right.\right.$ $\left.\left.y_{p}(t)\right]\right\} \exp (-d T)$. From Theorem 1 in [34], the pest-free solution $\left(x_{p}(t), y_{p}(t)\right)$ is locally stable if and only if $\left|\lambda_{1}\right|<1$, $\left|\lambda_{2}\right|<1$; that is,

$$
\mathrm{e}^{\int_{0}^{T}\left[a-c y_{p}(t)\right] \mathrm{d} t}<1, \quad\left|1-p_{2}^{\max }+\frac{p_{2}^{\max } \theta_{2}^{2}}{\theta_{2}+y_{p}(t)}\right| \mathrm{e}^{-d T}<1 .
$$

The latter inequality is trivial and the former gives $\operatorname{adT} /\left[c y^{*}\left(1-\mathrm{e}^{-d T}\right)\right] \leq 1$, which holds true due to $R_{0}^{1}<1$, so the local stability of $\left(x_{p}(t), y_{p}(t)\right)$ is proved.

We now focus on the global stability of the pest-free solution (12), and we should prove that $\bar{x}$ tends to be zero, and it follows that $\bar{y}$ tends to be zero; that is, $y(t)$ approximates to $y_{p}(t)$.

It follows from $\dot{\bar{y}} \geq-d \bar{y}$ and impulsive conditions that for any initial value $\left(\bar{x}_{0}, \bar{y}_{0}\right)$ we have $\bar{y} \geq \min \left(0, \bar{y}_{0}\right) \mathrm{e}^{-d T} \doteq \bar{y}_{m}(t)$. For convenience, we denote

$$
\begin{gathered}
\phi(x)=\frac{c x}{1+\omega x}, \quad f(x)=a x-b x^{2}, \\
\sup _{x \geq 0} \frac{f(x)}{\phi(x)}=\frac{(a \omega+b)^{2}}{4 b c \omega} \doteq M_{s}, \quad \sup _{x \geq 0} \frac{\phi(x)}{x}=c .
\end{gathered}
$$

In order to investigate the first equation of (14), let us consider the function $G(\bar{x})=\int_{x_{0}}^{\bar{x}} 1 / \phi(s) \mathrm{d} s$. It is easy to see that the function $G(\bar{x})$ is an increasing function from $\bar{x}=0$ as $\phi(x)>0$, where it goes to $-\infty$ since $\phi(\cdot)$ is locally Lipschitz on $\mathscr{R}^{+}$; then the pest population will die out eventually. Therefore, we write the $G(\bar{x})$ dynamics

$$
\begin{aligned}
\frac{\mathrm{d} G(\bar{x})}{\mathrm{d} t} & =\frac{1}{\phi(\bar{x})} \dot{\bar{x}}=\frac{f(\bar{x})}{\phi(\bar{x})}-\bar{y}(t)-y_{p}(t) \\
& \leq \frac{f(\bar{x})}{\phi(\bar{x})}-\bar{y}_{m}(t)-y_{p}(t) .
\end{aligned}
$$

In order to investigate the evolution of $G(x)$, we integrate $G$ between two successive pulse points, that is, the evolution of $G$ between the times $n T^{+}$and $(n+1) T$ for a given $n \in \mathcal{N}$

$$
\begin{aligned}
& G\left(\bar{x}\left((n+1) T^{+}\right)\right) \leq G\left(\bar{x}\left(n T^{+}\right)\right) \\
& \quad+\int_{n T^{+}}^{(n+1) T}\left[\frac{f(\bar{x}(s))}{\phi(\bar{x}(s))}-\bar{y}_{m}(s)-y_{p}(s)\right] \mathrm{d} s .
\end{aligned}
$$

We will now analyze how the pulse that takes place at time $(n+1) T$ impacts $G$, so

$$
\begin{aligned}
G & \left(\bar{x}\left((n+1) T^{+}\right)\right) \\
= & \int_{x_{0}}^{\bar{x}\left((n+1) T^{+}\right)} \frac{1}{\phi(s)} \mathrm{d} s \\
= & \int_{x_{0}}^{\bar{x}((n+1) T)} \frac{1}{\phi(s)} \mathrm{d} s+\int_{\bar{x}((n+1) T)}^{\bar{x}\left((n+1) T^{+}\right)} \frac{1}{\phi(s)} \mathrm{d} s \\
\leq & G(\bar{x}((n+1) T))+\int_{\bar{x}((n+1) T)}^{\bar{x}\left((n+1) T^{+}\right)} \frac{1}{c s} \mathrm{~d} s \\
\leq & G\left(\bar{x}\left(n T^{+}\right)\right)+\int_{n T^{+}}^{(n+1) T}\left[\frac{f(\bar{x}(s))}{\phi(\bar{x}(s))}-\bar{y}_{m}(s)-y_{p}(s)\right] \mathrm{d} s \\
& +\int_{\bar{x}((n+1) T)}^{\left[1-p_{1}(\bar{x}((n+1) T))\right] \bar{x}((n+1) T)} \frac{1}{c s} \mathrm{~d} s \\
= & G\left(\bar{x}\left(n T^{+}\right)\right)+\int_{n T^{+}}^{(n+1) T}\left[M_{s}-\bar{y}_{m}(s)-y_{p}(s)\right] \mathrm{d} s \\
& +\frac{\ln \left[1-p_{1}(x((n+1) T))\right]}{c}
\end{aligned}
$$




$$
\begin{aligned}
\leq & G\left(\bar{x}\left(n T^{+}\right)\right)+\int_{n T^{+}}^{(n+1) T}\left[M_{s}-\bar{y}_{m}(s)-y_{p}(s)\right] \mathrm{d} s \\
& +\frac{\ln \left(1-p_{1}^{\max }\right)}{c} .
\end{aligned}
$$

Defining $l$ as the integer part of $t / T$, we have

$$
\begin{aligned}
G( & \bar{x}(t))-G\left(x_{0}\right) \\
\leq & \int_{0}^{t}\left[M_{s}-\bar{y}_{m}(s)-y_{p}(s)\right] \mathrm{d} s+l \frac{\ln \left(1-p_{1}^{\max }\right)}{c} \\
= & -\int_{0}^{t} \bar{y}_{m}(s) \mathrm{d} s+\int_{l T}^{t}\left[M_{s}-y_{p}(s)\right] \mathrm{d} s \\
& +l \int_{0}^{T}\left[M_{s}-y_{p}(s)\right] \mathrm{d} s+l \frac{\ln \left(1-p_{1}^{\max }\right)}{c} \\
= & \frac{\min \left(0, \bar{y}_{0}\right)}{c}\left(\mathrm{e}^{-d t}-1\right)+\int_{l T}^{t}\left[M_{s}-y_{p}(s)\right] \mathrm{d} s \\
& +l \int_{0}^{T}\left[M_{s}-y_{p}(s)\right] \mathrm{d} s+l \frac{\ln \left(1-p_{1}^{\max }\right)}{c} .
\end{aligned}
$$

The first two terms are bounded due to the periodicity of $y_{p}(t)$ with period $T_{p}$. Note that $l \rightarrow \infty$ as $t \rightarrow \infty$. In fact, it suffices to have

$$
\int_{0}^{T}\left[M_{s}-y_{p}(s)\right] \mathrm{d} s+\frac{\ln \left(1-p_{1}^{\max }\right)}{c}<0
$$

to achieve this. It is better rewritten in the form

$$
\frac{c T M_{s}}{c \int_{0}^{T} y_{p}(s) \mathrm{d} s-\ln \left(1-p_{1}^{\max }\right)} \leq 1,
$$

which holds true due to $R_{0}^{2}<1$, so $\bar{x}(t)$ tends to be zero as $t \rightarrow \infty$, provided $R_{0}^{2}<1$.

Now we prove that $\bar{y}(t) \rightarrow 0$ as well. Since $\bar{x} \rightarrow 0$, so there exists a finite time $t_{s}$ such that $\phi(\bar{x}) \leq d / 2$ for $t>t_{s}$. Therefore we have

$$
\dot{\bar{y}}=\mu \phi(\bar{x})\left[\bar{y}+y_{p}(t)\right]-d \bar{y} \leq \mu \phi(\bar{x}) y_{p}(t)-\frac{d}{2} \bar{y}
$$

for $t>t_{s}$. It follows from $\bar{x} \rightarrow 0$ as $t \rightarrow \infty$ and the periodicity of $y_{p}(t)$ that we have $\phi(\bar{x}) y_{p}(t) \rightarrow 0$ as $t \rightarrow \infty$. Consequently, $\frac{p}{y} \rightarrow 0$ as $t \rightarrow \infty$. These results indicate that if $R_{0}^{2}<1$, then the pest-free periodic solution $\left(x_{p}(t), y_{p}(t)\right)$ is globally attractive, and in combination with the local stability criteria, we can conclude that the pest-free periodic solution $\left(x_{p}(t), y_{p}(t)\right)$ of the model (3) is globally stable. The proof is completed.

\section{B. The Proof of Theorem 4}

In order to apply the bifurcation theory of [34], we make the following calculations:

$$
d_{0}^{\prime}=1-\mathrm{e}^{\int_{0}^{T_{0}}\left[a-c y_{p}(\xi)\right] \mathrm{d} \xi} .
$$

If $d_{0}^{\prime}=0, T_{0}$ satisfies the following condition:

$$
\frac{c y^{*}\left(1-\mathrm{e}^{-d T_{0}}\right)}{a d T_{0}}=1,
$$

which indicates that there exists a $T_{0}$ such that $R_{0}^{1}=1$ and the pest-free periodic solution $\sigma=\left(0, y_{p}(t)\right)$ loses its stability.

Further,

$$
\begin{aligned}
& \frac{\partial \Phi_{1}\left(T_{0}, X_{0}\right)}{\partial y}=\mathrm{e}^{-d T_{0}}>0 ; \\
& \frac{\partial \Phi_{2}\left(T_{0}, X_{0}\right)}{\partial x}=\mathrm{e}^{\int_{0}^{T_{0}}\left[a-c y_{p}(\xi)\right] \mathrm{d} \xi}>0 ; \\
& \frac{\partial \Phi_{1}\left(T_{0}, X_{0}\right)}{\partial x}=\int_{0}^{T_{0}} \mu c y_{p}(v) \cdot \mathrm{e}^{-d\left(T_{0}-v\right)} \\
& \cdot \mathrm{e}^{\int_{0}^{v}\left[a-c y_{p}(\xi)\right] \mathrm{d} \xi} \mathrm{d} v \doteq A_{4}>0 ; \\
& d_{0}^{\prime}=1-\mathrm{e}^{\int_{0}^{T_{0}}\left[a-c y_{p}(\xi)\right] \mathrm{d} \xi} ; \\
& a_{0}^{\prime}=1-A_{3} \mathrm{e}^{-d T_{0}} ; \\
& A_{3} \doteq 1-p_{\text {max }}^{2}+\frac{p_{\text {max }}^{2} \theta_{2}^{2}}{\left[y_{p}\left(T_{0}\right)+\theta_{2}\right]^{2}} ; \\
& b_{0}^{\prime}=1-A_{3} A_{4} ; \\
& \frac{\partial^{2} \Phi_{2}\left(T_{0}, X_{0}\right)}{\partial x \partial y}=-c T_{0} \cdot \mathrm{e}^{\int_{0}^{T_{0}}\left[a-c y_{p}(\xi)\right] \mathrm{d} \xi}<0 ; \\
& \frac{\partial^{2} \Phi_{2}\left(T_{0}, X_{0}\right)}{\partial x^{2}} \\
& =-2 T_{0} \cdot\left[b+c \omega y_{p}(v)\right] \cdot e^{\int_{0}^{T_{0}}\left[a-c y_{p}(\xi)\right] \mathrm{d} \xi} \\
& -c \int_{0}^{T_{0}}\left\{\mathrm{e}^{\int_{v}^{T_{0}}\left[a-c y_{p}(\xi)\right] \mathrm{d} \xi}\right. \\
& \cdot \int_{0}^{v}\left\{\mu c y_{p}(\theta) \cdot \mathrm{e}^{-d(v-\theta)}\right. \\
& \left.\left.\cdot e^{\int_{0}^{\theta}\left[a-c y_{p}(\xi)\right] \mathrm{d} \xi}\right\} \mathrm{d} \theta\right\} \mathrm{d} v \\
& \doteq A_{5}<0 \text {; } \\
& \frac{\partial^{2} \Phi_{2}\left(T_{0}, X_{0}\right)}{\partial x \partial \bar{T}}=\left[a-c y_{p}\left(T_{0}\right)\right] \cdot \mathrm{e}^{\int_{0}^{T_{0}}\left[a-c y_{p}(\xi)\right] \mathrm{d} \xi} ; \\
& \frac{\partial \Phi_{1}\left(T_{0}, X_{0}\right)}{\partial \bar{T}}=-d y^{*}\left(T_{0}\right) \mathrm{e}^{-d T_{0}} ; \\
& M=\left\{-c d T_{0} y^{*}\left(T_{0}\right) \cdot \frac{A_{3} \mathrm{e}^{-d T_{0}}}{1-A_{3} \mathrm{e}^{-d T_{0}}}\right. \\
& \left.-\left[a-c y_{p}\left(T_{0}\right)\right]\right\} \cdot \mathrm{e}^{\int_{0}^{T_{0}}\left[a-c y_{p}(\xi)\right] \mathrm{d} \xi} ;
\end{aligned}
$$




$$
\begin{aligned}
N= & 2 p_{1}^{\max } \theta_{1}^{-1} \cdot \mathrm{e}^{2 \int_{0}^{T_{0}}\left[a-c y_{p}(\xi)\right] \mathrm{d} \xi} \\
& -2 c T_{0} \cdot \frac{1-A_{3} A_{4}}{1-A_{3} \mathrm{e}^{-d T_{0}}} \cdot \mathrm{e}^{\int_{0}^{T_{0}}\left[a-c y_{p}(\xi)\right] \mathrm{d} \xi}-A_{5} .
\end{aligned}
$$

It follows from $M N<0$ that we get the condition $C_{1}$ or $C_{2}$; that is, if the parameters satisfy $C_{1}$ or $C_{2}$, then model (3) has a supercritical branch at $T_{0}$. The proof is completed.

\section{Conflict of Interests}

The authors declare that there is no conflict of interests regarding the publication of this paper.

\section{Acknowledgments}

This work is supported by the National Natural Science Foundation of China (NSFC: 11171199, 11371030, and 11301320) and the Fundamental Research Funds for the Central Universities (GK201305010, GK201401004). The authors would like to thank the editor and the referees for their careful reading of the original paper and many valuable comments and suggestions that greatly improved the presentation of this paper.

\section{References}

[1] P. DeBach, Biological Control of Insect Pests and Weeds, Rheinhold, New York, NY, USA, 1964.

[2] H. I. Freedman, "Graphical stability, enrichment, and pest control by a natural enemy," Mathematical Biosciences, vol. 31, no. 3-4, pp. 207-225, 1976.

[3] L. E. Caltagirone and R. L. Doutt, "The history of the vedalia beetle importation to California and its impact on the development of biological control," Annual Review of Entomology, vol. 34, pp. 1-16, 1989.

[4] V. Stern, R. Smith, R. Van den Bosch, and K. Hagen, "The integrated control concept," Hilgardia, vol. 29, no. 2, pp. 81-101, 1959.

[5] R. Van den Bosch, The Pesticide Conspiracy, University of California Press, Berkeley, Calif, USA, 1989.

[6] J. Van Lenteren, "Environmental manipulation advantageous to natural enemies of pests," in Integrated Pest Management, V. Delucchi, Ed., pp. 123-166, Parasitis, Geneva, Switzerland, 1987.

[7] J. Van Lenteren, "Integrated pest management in protected crops," in Integrated Pest Management, D. Dent, Ed., pp. 311-320, Chapman \& Hall, London, UK, 1995.

[8] Y. Xiao and F. Van Den Bosch, "The dynamics of an ecoepidemic model with biological control," Ecological Modelling, vol. 168, no. 1-2, pp. 203-214, 2003.

[9] H. J. Barclay, "Models for pest control using predator release, habitat management and pesticide release in combination," Journal of Applied Ecology, vol. 19, no. 2, pp. 337-348, 1982.

[10] D. Bainov and P. Simeonov, Impulsive Differential Equations: Periodic Solutions and Applications, Longman Scientific \& Technical, Harlow, UK, 1993.

[11] V. Lakshmikantham, D. Bainov, and P. Simeonov, Theory of Impulsive Differential Equations, World Scientific, Singapore, 1989.
[12] S. Tang and L. Chen, "Modelling and analysis of integrated pest management strategy," Discrete and Continuous Dynamical Systems B, vol. 4, no. 3, pp. 759-768, 2004.

[13] S. Tang, Y. Xiao, and D. Clancy, "New modelling approach concerning integrated disease control and cost-effectivity," Nonlinear Analysis: Theory, Methods \& Applications, vol. 63, no. 3, pp. 439-471, 2005.

[14] S. Tang, Y. Xiao, L. Chen, and R. A. Cheke, "Integrated pest management models and their dynamical behaviour," Bulletin of Mathematical Biology, vol. 67, no. 1, pp. 115-135, 2005.

[15] S. Tang, Y. Xiao, and R. A. Cheke, "Multiple attractors of hostparasitoid models with integrated pest management strategies: eradication, persistence and outbreak," Theoretical Population Biology, vol. 73, no. 2, pp. 181-197, 2008.

[16] S. Tang, G. Tang, and R. A. Cheke, "Optimum timing for integrated pest management: modelling rates of pesticide application and natural enemy releases," Journal of Theoretical Biology, vol. 264, no. 2, pp. 623-638, 2010.

[17] J. Liang, S. Tang, and R. A. Cheke, "An integrated pest management model with delayed responses to pesticide applications and its threshold dynamics," Nonlinear Analysis: Real World Applications, vol. 13, no. 5, pp. 2352-2374, 2012.

[18] S. Tang, J. Liang, Y. Tan, and R. A. Cheke, “Threshold conditions for integrated pest management models with pesticides that have residual effects," Journal of Mathematical Biology, vol. 66, no. 1-2, pp. 1-35, 2013.

[19] P. Georgescu, H. Zhang, and L. Chen, "Bifurcation of nontrivial periodic solutions for an impulsively controlled pest management model," Applied Mathematics and Computation, vol. 202, no. 2, pp. 675-687, 2008.

[20] B. Liu, L. Chen, and Y. Zhang, "The dynamics of a preydependent consumption model concerning impulsive control strategy," Applied Mathematics and Computation, vol. 169, no. 1, pp. 305-320, 2005.

[21] B. Williams, J. Nichols, and M. Conroy, Analysis and Management of Animal Populations, Academic Press, San Diego, 2002.

[22] M. J. Reynolds-Hogland, J. S. Hogland, and M. S. Mitchell, "Evaluating intercepts from demographic models to understand resource limitation and resource thresholds," Ecological Modelling, vol. 211, no. 3-4, pp. 424-432, 2008.

[23] M. J. Reynolds-Hogland, L. B. Pacifici, and M. S. Mitchell, "Linking resources with demography to understand resource limitation for bears," Journal of Applied Ecology, vol. 44, no. 6, pp. 1166-1175, 2007.

[24] S. Blower and H. Dowlatabadi, "Sensitivity and uncertainty analysis of complex-models of disease transmission? An HIV model, as an example," International Statistical Review, vol. 62, no. 2, pp. 229-243, 1994.

[25] S. Marino, I. B. Hogue, C. J. Ray, and D. E. Kirschner, "A methodology for performing global uncertainty and sensitivity analysis in systems biology," Journal of Theoretical Biology, vol. 254, no. 1, pp. 178-196, 2008.

[26] A. Lotka, Elements of Physical Biology, William and Wilkins, Baltimore, Md, USA, 1925.

[27] V. Volterra, "Variazioni e fluttuazioni del numero d'individui in specie animali conviventi," Memorie della Regia Accademia Nazionale dei Lincei, vol. 2, no. 5, pp. 31-113, 1926.

[28] C. Holling, "The functional response of predator to prey density and its role in mimicry and population regulation," Memoirs of the Entomological Society of Canada, vol. 97, no. S45, pp. 1-60, 1965. 
[29] X. Liu and L. Chen, "Complex dynamics of Holling type II Lotka-Volterra predator-prey system with impulsive perturbations on the predator," Chaos, Solitons and Fractals, vol. 16, no. 2, pp. 311-320, 2003.

[30] B. Liu, Z. Teng, and L. Chen, "Analysis of a predator-prey model with Holling II functional response concerning impulsive control strategy," Journal of Computational and Applied Mathematics, vol. 193, no. 1, pp. 347-362, 2006.

[31] A. Hill, "The possible effects of the aggregation of the molecules of haemoglobin on its dissociation curves," The Journal of Physiology, vol. 40, pp. 404-407, 1910.

[32] P. Cull, "Local and global stability for population models," Biological Cybernetics, vol. 54, no. 3, pp. 141-149, 1986.

[33] M. D. McKay, R. J. Beckman, and W. J. Conover, "A comparison of three methods for selecting values of input variables in the analysis of output from a computer code," Technometrics, vol. 21, no. 2, pp. 239-245, 1979.

[34] A. Lakmeche and O. Arino, "Bifurcation of non trivial periodic solutions of impulsive differential equations arising chemotherapeutic treatment," Dynamics of Continuous, Discrete and Impulsive Systems, vol. 7, no. 2, pp. 265-287, 2000.

[35] S. Tang and L. Chen, "Multiple attractors in stage-structured population models with birth pulses," Bulletin of Mathematical Biology, vol. 65, no. 3, pp. 479-495, 2003. 


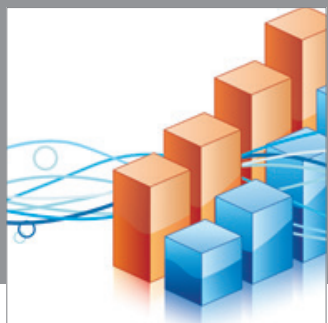

Advances in

Operations Research

mansans

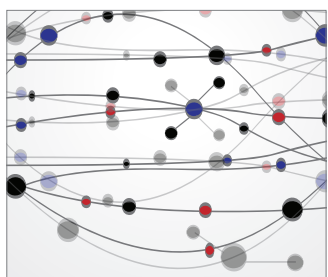

The Scientific World Journal
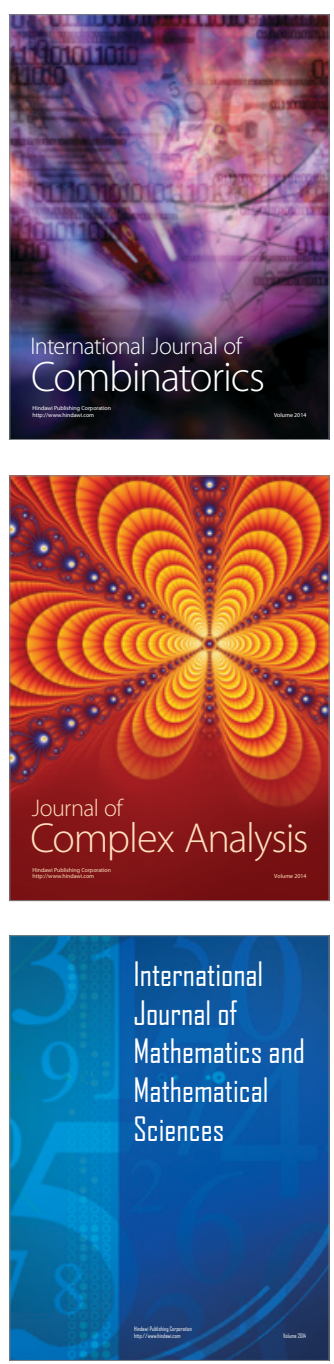
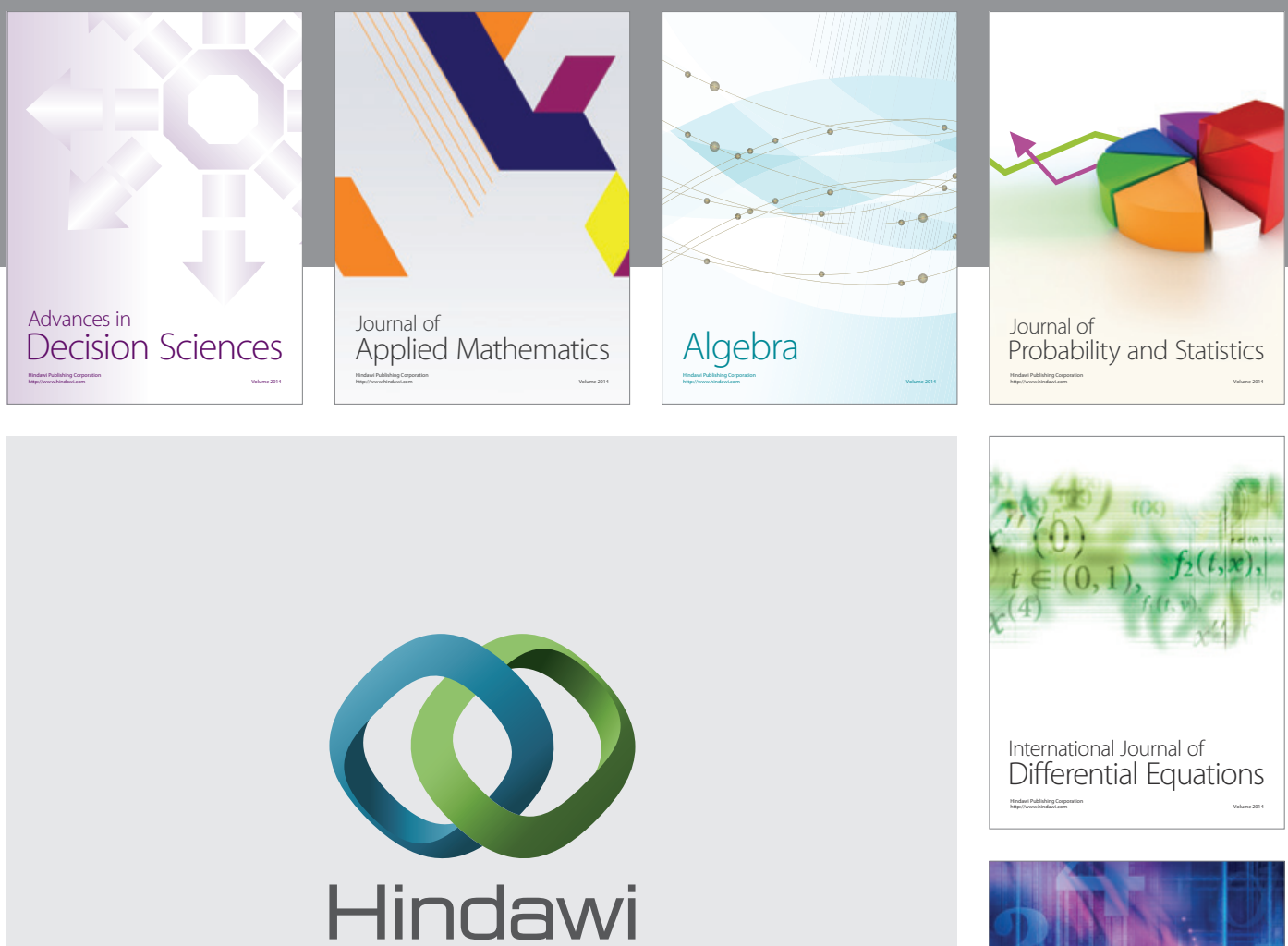

Submit your manuscripts at http://www.hindawi.com
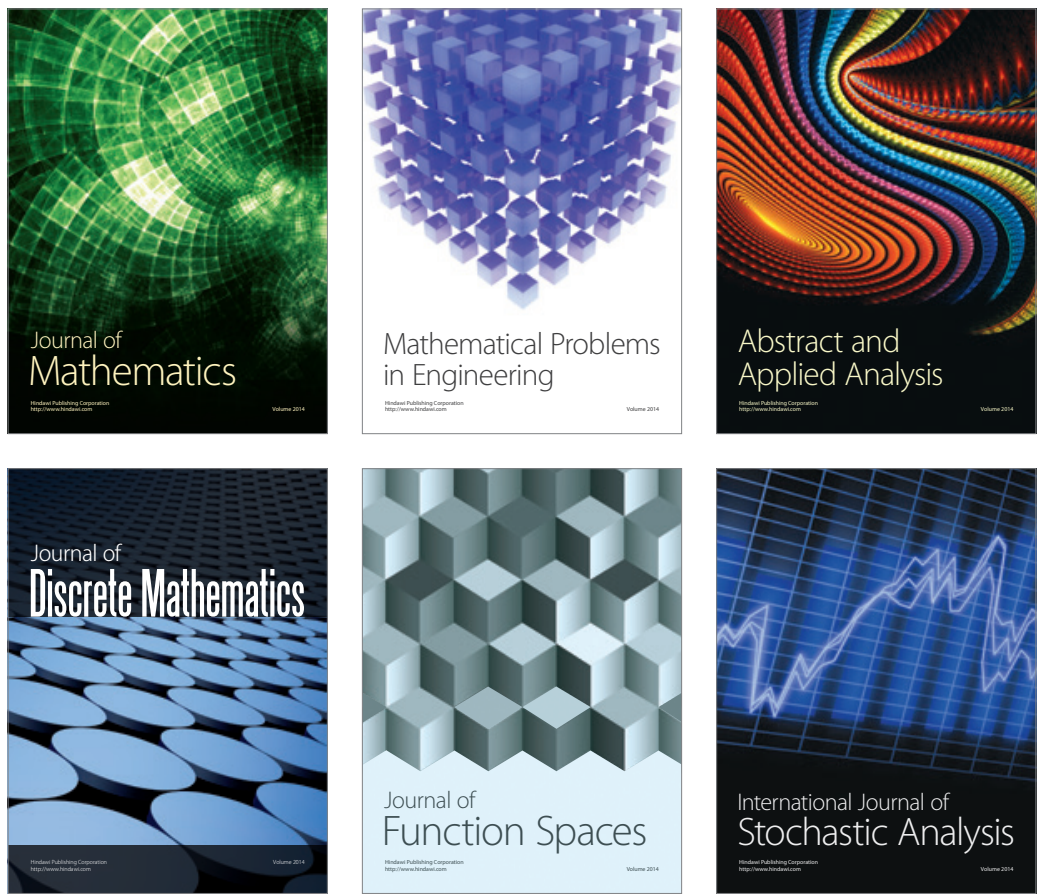

Journal of

Function Spaces

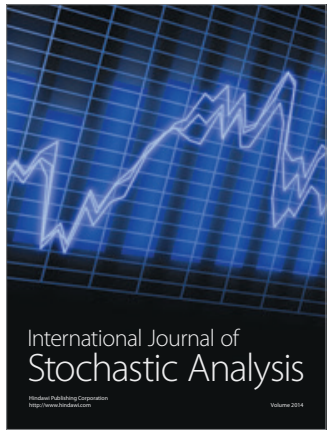

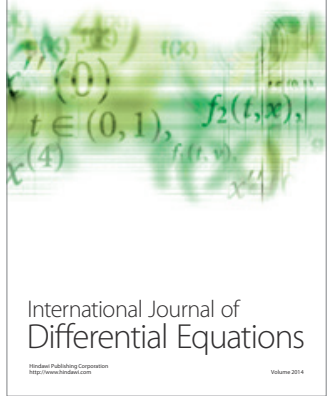
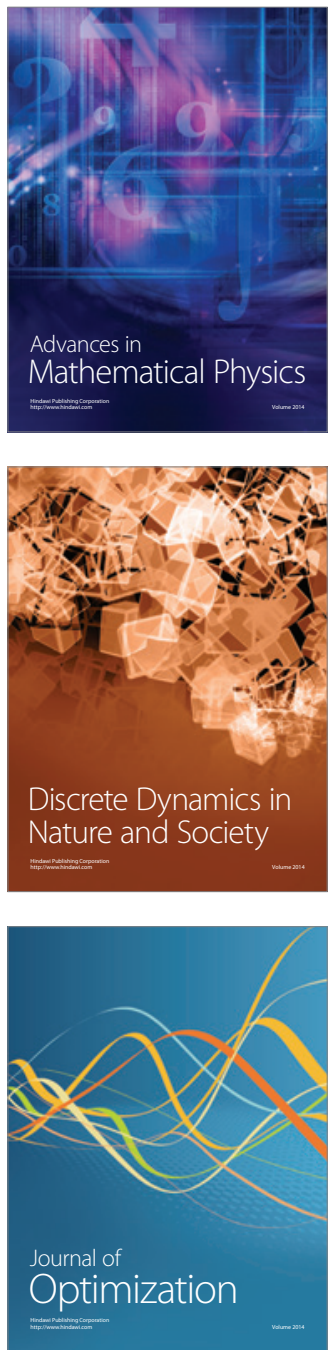